\title{
Critical Challenges for Requirement Implementation in Global Software Development: A Systematic Literature Review Protocol with Preliminary Results
}

\author{
Zahid Ali \\ University of Engineering \& Technology, \\ Peshawar, University of Swat \\ Khyber Pakhtunkhwa, Pakistan
}

\author{
Muhammad Yaseen \\ Engineering Research \& IT Services \\ Provider (Pvt) Ltd \\ Peshawar, Pakistan
}

\begin{abstract}
Context: Successful requirement engineering (RE) leads to successful delivery of software. There exist a lot of challenges during RE process especially in Global Software Development (GSD). The aim of this study is to find challenges during RE in context of GSD.
\end{abstract}

Objective: The objective is to find and analyze the challenges in RE process.

Method: Systematic Literature Review (SLR) is used as research method to achieve the goals.

Result: SLR protocol is developed. Expected output of this study is to list out all challenges which the stakeholders are facing in implementing RE in the context of GSD through SLR. As a result of SLR protocol, 71 papers are identified.

\section{Keywords}

Systematic Literature Review; Requirement engineering; Requirement Implementation; Global Software Development; Systematic Literature Review Protocol.

\section{INTRODUCTION}

$\mathrm{RE}$ is a systematic way of collecting requirements from user in a proper way by applying various elicitation techniques [1, 2]. RE consist of four phases [17]. Requirement elicitation is the first phase in which we collect requirements from users, requirement analysis phase is the second phase in which user requirements are analyzed, In specification phase, final list of functional requirements are specified in document known as SRS. In validation phase, requirements are validated against SRS. The quality of final deliver software is totally depended on proper requirement implementation [3][18]. The more errors and bugs remain in this phase, the more is the chances of failure of the software and the more it is expensive to fix it latterly on [3]. RE needs more attention and much effort is required. The RE is difficult when implemented it locally but in case of GSD the case is worst because there exists some challenges like culture difference, physical and geographical change, language and terminology difference, lack of face to face conversation and time zone difference [3-5]. Their exists communication and coordination problems during requirement elicitation in GSD [6]. To overcome the challenges, some practices and success factors are Needed [7]19]. We need to address these challenges systematically and should analyze these challenges through company size, different continents, decade and experimental methodologies. Our objective is to find critical challenges in path of RE process in GSD through SLR. SLR protocol is developed and discussed here in detail. After protocol development and SLR process will be conducted.

\section{BACKGROUND STUDY}

According to Yvonne Hsieh [3] culture diversity causes coordination problems and is a big challenge because people belongs to different cultures and have different explanations and translation of requirements. Coordination and collaboration is a big success factor in GSD. According to Daniela Damian [4] the cause of improper conversation is due to time difference because most of the time if there is a night in one country the other country has day so difficult to communicate. According to Paula Laurent [1] lack of an appropriate technical infrastructure prohibit inter-site collaboration. Face to face communication is the best way to resolve issues and misunderstandings but in GSD the big challenge is that teams are physically separated. According to Vibha Sinha [8] requirement change management is very difficult because it needs proper negotiations and discussion which is difficult without face to face meeting. Common understanding of requirements requires integration with communicating environment. According to Barkha Javed [9] knowledge sharing is big problem during RE process due to culture diversity and terminology difference and there is a lack of proper and effective communication due to which knowledge management also becomes difficult. Paolo Tell [10] also discuss the issues arises due to lack of communication which cause improper negotiations in time. According to Daniela E. Damian [11] building trust in GSD is very difficult because there are some factors which are necessary for trust building like face to face meeting and proper discussions but due to distance and culture diversity building trust becomes challenge. Coordination and collaboration is also a big source of trust building. The author says that language and terminology difference is a big challenge and it affects requirement elicitation process because this phase of RE process requires direct communication with clients to gather requirements and transfer knowledge and this factor causes difficulty. According to Nosheen Sabahat [12] proper global project management is necessary to tackle all the challenges and to bring coordination among the team members. Brian Berenbach [13] also discusses the importance of role of project management in GSD and according to him one of the observed issue was lack of effective leadership. According to S. Arun Kumar [14] requirement management is a big challenge in GSD and it needs change management frameworks and models which can solve these problems. The author discusses the challenges which arise due to low involvement of customers in GSD.

S. Sakthivel [15] says that experience with new technologies and the complexities arises from technologies are considered as barrier in path of negotiations and discussing requirements. 
The author further says that cost of infrastructure is also an issue for client's side mostly to discuss the requirements. Besides understanding and selection of proper RE process is not easy task so it needs to focus more.

\section{RESEARCH METHOD}

SLR is different from ordinary literature review as it is more planned and methodically executed because in ordinary literature review we randomly search publications without following any systematic and planned procedure. Figure 1 shows step by step execution of research methodology. Other authors also used the same method [16] [20] [21].

\subsection{SLR Protocol Developments}

Before conducting the systematic review, review protocol was developed. A pre-defined protocol increases the hardship and iteration of the review. Procedures and review plan is specified through SLR. The various stages of SLR process are aim and need, research questions, search string, involvement and removing criteria, form of data extraction and arrange data from papers. Fig1 shows SLR protocol developments stages.

\subsection{Research Questions}

The following 5 questions were arises while conducting SLR.

RQ1. What are the challenges, as discussed in the literature, elaborated from software vendor's view in implementing requirements engineering processes throughout organizations in context GSD?Based on objective and the above Research Question some further questions arises like

RQ2. Is there any relationship between these challenges and size of organization in GSD?

RQ3.How these challenges vary from continent to continent in GSD?

RQ4.How these challenges are related to different research methods in GSD?

RQ5. What is the impact of changing period on these factors in GSD?

We will make search string for the RQ1 only, papers selected based on RQ1 will be analyzed for answering the rest of questions.

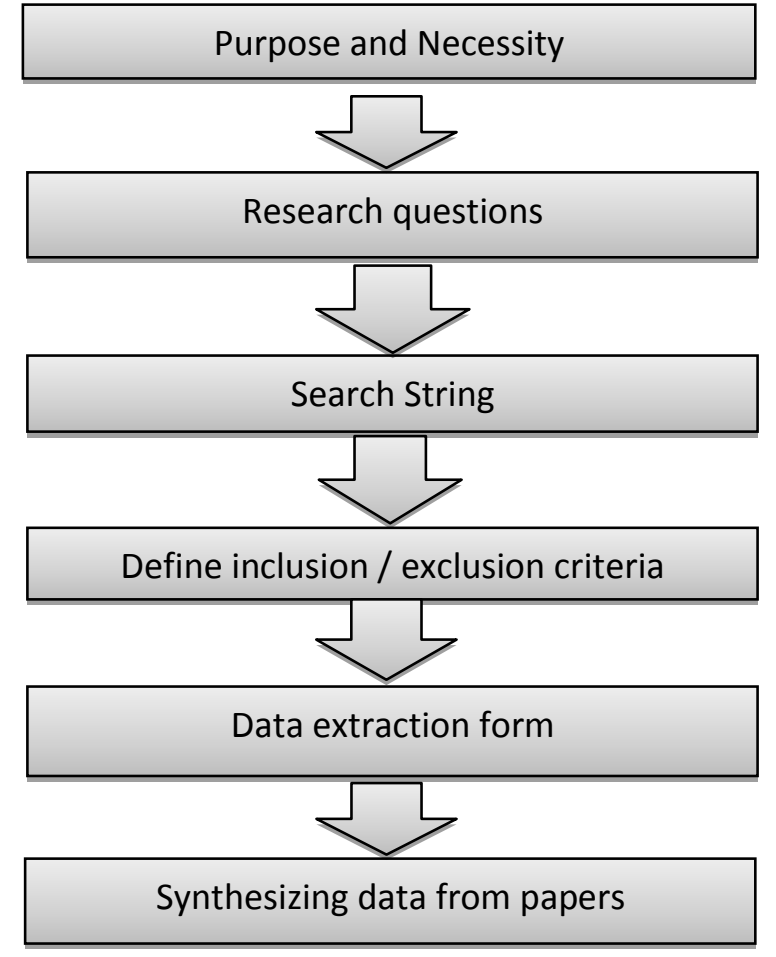

Fig 1.Development process for the SLR Protocol

\subsection{Making of Search Terms}

The following factors should be consider while conducting SLR.

1. People: Clients and Vendors included in GSD

2. Interference: challenges in requirement implementation.

3. Outcomes of relevance: Better implementation of RE in GSD.

Research questions contain the following above information's.

RQ1. [What are the challenges, as discussed in the literature] "Interference", elaborated from software vendor's view in [implementing requirements engineering] "Outcome" processes throughout organizations in context [GSD] "People". ?

\subsection{Search Strategy}

It include the following sections.

- $\quad$ Range of search ( time and space)

- Method for searching

- Electronic data sources used

- $\quad$ Strings for the search

- Validation of search

- Documentation of the search

- Management of search result 


\subsubsection{Range of search (time and space)}

Refer to research questions we will search for all published literature with no bound and limit on any time (years).

\subsubsection{Method for searching}

There are two search method automatic searches or manual search. Search string is executed on search engines of electronic data sources in automatic searching while in manual searching search is done by browsing through specific journals or conference proceedings.

\subsubsection{Electronic data sources used}

- Google scholar

- $\quad$ Science Direct

- $\quad$ Springer link

- Acm portal

- IEEE Xplore

\subsubsection{Strings for the search}

Search string is split into three types of sub search strings.

A. Preliminary search string: This will be initial string which will help in preliminary search.

B. Big search string: Using Boolean operators and by combination of major terms and its equivalents we can obtain this string.

C. Smaller sub search string: As some of libraries do not get long string so we will divide it into smaller strings and will do the independent search for each string

\subsubsection{Search terms identification}

The below steps (strategy for searching) are used for making of search terms.

Step1: Major terms derivation: For the derivation of major terms use the research questions, by identifying people, medium and outcome.

Step2: Find the substitute spellings and synonyms for these major terms

Step3: Find out the key words in any related paper;

Step4: Use Boolean Operators for combination if the library allows. Use "OR" in case of substitute spellings and synonyms and use "AND" in case of combination of major terms.

\subsubsection{Documentation of the search}

Proper documentation of search results is necessary, and the following data will be listed:

- Database name

- Strategy for the search

- $\quad$ Phase of the search

- Search date

- No of publications found

- No of publication chosen

- Decision of introductory chosen

- Decision of final chosen

-

\subsection{Selection of publications}

Fig 2 shows the detail of publication selection process.

- Involvement Criteria

- Removing Criteria

- Determination of Publication Quality

\subsubsection{Inclusion Criteria}

Entry criteria will be used to limit the number of papers which are retrieved by applying search strings and which are included for final data selection. The following are some inclusion criteria

- Papers written in English only are acceptable

- Papers which are related to RE in GSD only

- $\quad$ Papers which discuss the challenges only during requirement implementation in GSD.

- $\quad$ Studies that are related to RE only but they are fitted in GSD also.

\subsubsection{Exclusion criteria}

On the basis of removing criteria we decide which paper will be removed from the final list. The following are some exclusion criteria on base of which we will exclude papers from selected ones

- Studies which are not related to our Research questions

- Studies that do not discuss RE in GSD

- $\quad$ Studies that do not discuss challenges during RE in GSD.

- $\quad$ Papers belong to GSD but they don't discuss RE

- Papers that discuss RE but don't fit in GSD.

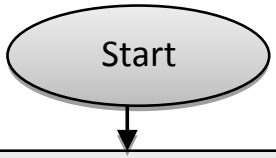

Search for papers from relevant journals and conferences

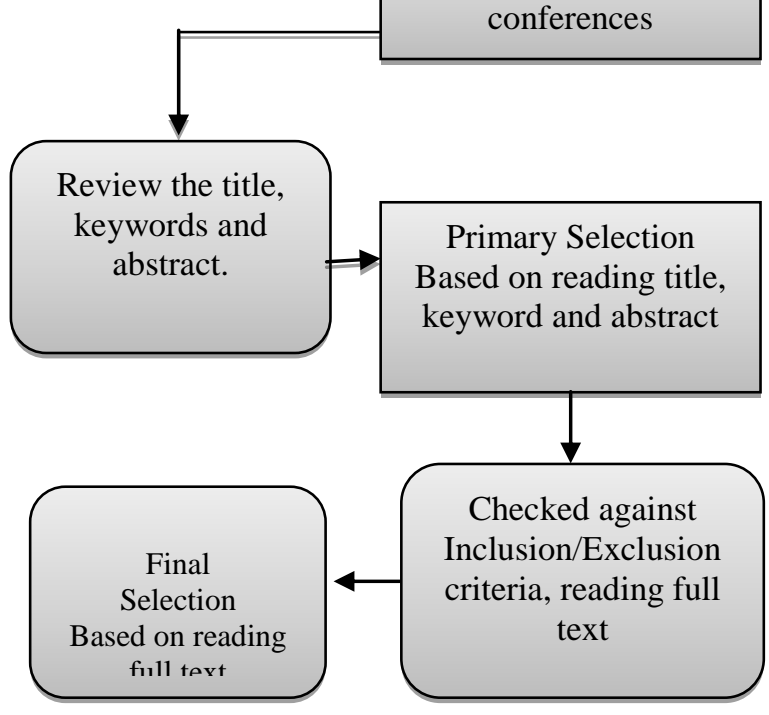

Figure 2. Publication Selection process 


\subsection{Strategy of data extraction}

Data extraction consist of the following section.

\subsubsection{Primary study data}

The data Extracted from publications will contain the following.

\subsubsection{Data extraction process}

One person will do the extraction for review. Secondary person can provide the guidance if he find problems in data extraction.

\subsubsection{Data synthesis}

We will synthesize the extracted data in SLR which give answers of the research questions. The following data will be synthesizes.

- Date of review

- Publication details (Title, Authors, Reference)

- Sample Population

- $\quad$ Company size (small, medium, large)

- Location of the Analysis (continent)

- Publication year

- challenges in requirement implementation in GSD

- Publication Quality Description

\section{RESULTS}

Total of 71 papers were selected after applying inclusion and exclusion criteria as discussed. The SLR protocol was executed as planned. All papers are mention in appendix list below.

Table 1: final selected papers

\begin{tabular}{|c|c|c|c|}
\hline Resource & $\begin{array}{c}\text { Total } \\
\text { Results }\end{array}$ & $\begin{array}{c}\text { Primary } \\
\text { selection }\end{array}$ & $\begin{array}{c}\text { Final } \\
\text { selection }\end{array}$ \\
\hline IEEE explore & 1139 & 60 & 36 \\
\hline ACM & 1200 & 15 & 07 \\
\hline Science Direct & 2735 & 23 & 06 \\
\hline Springer Link & 500 & 18 & 06 \\
\hline Others & 2500 & 55 & 16 \\
\hline Total & 8074 & 171 & 71 \\
\hline
\end{tabular}

\section{CONCLUSION}

SLR protocol were developed and discussed in detail. The next step will be to apply this protocol and do SLR process. After SLR we will identify list of challenges and will mark some factors as more critical and will make analysis of the factors. In future we will find practices needed to implement RE in GSD successfully. The end goal is to make Requirement implementation model which will address the challenges and its solutions and practices in the context of GSD.

\section{REFERENCES}

[1] P. Laurent. (2010), "Globally Distributed Requirements Engineering.," presented at ICGSE.
[2] A. Ahmad, A. Shahzad. (2011). V. K. Padmanabhuni, A. Mansoor, S. Joseph, and Z. Arshad, "Requirements prioritization with respect to Geographically Distributed Stakeholders," presented at Computer Science and Automation Engineering (CSAE).

[3] Y. Hsieh, (2006). "Culture and shared understanding in distributed requirements engineering," presented at Global Software Engineering conference.

[4] D. Damian,(2007) "Stakeholders in global requirements engineering: Lessons learned from practice," Software, IEEE, vol. 24, pp. 21-27.

[5] C. Lescher and B. Brugge, (2009). "Global requirements engineering: decision support for globally distributed projects," presented at Global Software Engineering, 2009. ICGSE. Fourth IEEE International Conference.

[6] I. H. de Farias Junior, R. R. de Azevedo,(2012). H. P. de Moura, and D. S. Silva, "Elicitation of Communication Inherent Risks in Distributed Software Development," presented at Global Software Engineering Workshops (ICGSEW), IEEE Seventh International Conference.

[7] J. M. Bhat, M. Gupta, and S. N. Murthy, (2006). "Overcoming requirements engineering challenges: Lessons from offshore outsourcing," Software, IEEE, vol. 23 , pp. 38-44.

[8] V. Sinha, B. Sengupta, and S. Chandra, (2006) "Enabling collaboration in distributed requirements management," Software, IEEE, vol. 23, pp. 52-61.

[9] B. Javed and S. S. Minhas, (2010) "Process Support for Requirements Engineering Activities in Global Software Development: A Literature Based Evaluation," presented at Computational Intelligence and Software Engineering (CiSE), International Conference.

[10] P. Tell and M. A. Babar, (2011) "Requirements for an infrastructure to support activity-based computing in global software development," presented at Global Software Engineering Workshop (ICGSEW), Sixth IEEE International Conference.

[11] D. E. Damian and D. Zowghi, (2003). "RE challenges in multi-site software development organisations," Requirements engineering, vol. 8, pp. 149-160.

[12] N. Sabahat, F. Iqbal, F. Azam, and M. Y. Javed, (2010) "An iterative approach for global requirements elicitation: A case study analysis," presented at Electronics and Information Engineering (ICEIE), International Conference.

[13] B. Berenbach, (2006) "Impact of organizational structure on distributed requirements engineering processes: lessons learned," presented at Proceedings of the international workshop on Global software development for the practitioner.

[14] S. A. Kumar and T. A. Kumar, (2011) "Study the Impact of Requirements Management Characteristics in Global Software Development Project: An Ontology Based Approach," International Journal of Software Engineering and Application, vol. 2.

[15] S. Sakthivel, (2007) "Managing risk in offshore systems development," Communications of the ACM, vol. 50, pp. 69-75. 
[16] S. Keele, (2007) "Guidelines for performing systematic literature reviews in software engineering," in Technical report, Ver. 2.3 EBSE Technical Report. EBSE.

[17] M. Yaseen, A. Mustapha, and N. Ibrahim An Approach for Managing Large-Sized Software Requirements During Prioritization', no. 1, pp. 98-103, 2019.

[18] M. Yaseen, A. Mustapha, and N. Ibrahim, "Requirements prioritization nd using Iteration Model for successful Implementation of Requirements", Int. J. Adv. Comput. Sci. Appl., vol. 10, no.1, pp.121-127, 2019.

[19] M. Yaseen and U. Farooq, "Requirement Elicitation Model (REM) in the Context of Global Software Development", Glob. J. Comput. Sci. Technol., vol. 7, no. 3, pp. 303-308, 2018

[20] M. Yaseen, S. Baseer, and S. Sherin, "Critical challenges for requirement implementation in context of global software development: A systematic literature review" ICOSST 2015 - 2015 Int. Conf. Open Source Syst. Technol. Proc., vol. 9, no. 6, pp. 120-125, 2016

[21] M. Yaseen, S. Ali, Abdullah, N. Ullah "An Improved Framework for Requirement Implementation in the context of Global Software Development: A Systematic Literature Review Protocol", International Journal of Database Theory and Application Vol.9, No.6 (2016), pp.161-170

\section{APPENDIX}

\section{List of Papers}

W. J. Lloyd, M. B. Rosson, and J. D. Arthur, "Effectiveness of elicitation techniques in distributed requirements engineering," presented at Requirements Engineering, 2002. Proceedings. IEEE Joint International Conference on, 2002.

Y. Hsieh, "Culture and shared understanding in distributed requirements engineering," presented at Global Software Engineering, 2006. ICGSE'06. International Conference on, 2006.

D. Damian, "Stakeholders in global requirements engineering: Lessons learned from practice," Software, IEEE, vol. 24, pp. $21-27,2007$

P. Laurent, "Globally Distributed Requirements Engineering.," presented at ICGSE, 2010.

D. Damian, "An empirical study of requirements engineering in distributed software projects: is distance negotiation more effective?," presented at Software Engineering Conference, 2001. APSEC 2001. Eighth Asia-Pacific, 2001.

C. Lescher and B. Brugge, "Global requirements engineering: decision support for globally distributed projects," presented at Global Software Engineering, 2009. ICGSE 2009. Fourth IEEE International Conference on, 2009.

V. Sinha, B. Sengupta, and S. Chandra, "Enabling collaboration in distributed requirements management," Software, IEEE, vol. 23, pp. 52-61, 2006.

A. Lopez, J. Carrillo-de-Gea, and A. Toval, "Risks and safeguards for the requirements engineering process in global software development," presented at Global Software Engineering, 2009. ICGSE 2009. Fourth IEEE International Conference on, 2009.

A. Hoffmann and C. Lescher, "Collaboration and intercultural issues on requirements: Communication, understanding and softskills (circus)," presented at Requirements: Communication, Understanding and Softskills, 2009 Collaboration and Intercultural Issues on, 2009.

A. A. Khan, S. Basri, and P. Dominic, "A propose framework for requirement Change Management in Global Software Development," presented at Computer \& Information Science (ICCIS), 2012 International Conference on, 2012.

C. Solis and N. Ali, "Distributed requirements elicitation using a spatial hypertext wiki," presented at Global Software Engineering (ICGSE), 2010 5th IEEE International Conference on, 2010.

K. Sugawara, Y. Manabe, C. Moulin, and J.-P. Barthes, "Design assistant agents for supporting requirement specification definition in a distributed design team," presented at Computer Supported Cooperative Work in Design (CSCWD), 2011 15th International Conference on, 2011.

P. Tell and M. A. Babar, "Requirements for an infrastructure to support activity-based computing in global software development," presented at Global Software Engineering Workshop (ICGSEW), 2011 Sixth IEEE International Conference on, 2011.

M. Humayun and C. Gang, "Investigating the role of organizational structure in developing shared understanding of requirements within GSD," presented at Multitopic Conference (INMIC), 2012 15th International, 2012.

W. Hussain, J. Buchan, and T. Clear, "Managing Requirements in Globally Distributed COTS Customization," presented at Global Software Engineeering Workshops (ICGSEW), 2014 IEEE International Conference on, 2014.

G. N. Aranda, A. VizcaÃ-no, A. Cechich, and M. Piattini, "Strategies to minimize problems in global requirements elicitation," CLEI electronic journal, vol. 11, 2008.

[D. E. Damian and D. Zowghi, "The impact of stakeholders' geographical distribution on managing requirements in a multi-site organization," presented at Requirements Engineering, 2002. Proceedings. IEEE Joint International Conference on, 2002.

D. E. Damian and D. Zowghi, "RE challenges in multi-site software development organisations," Requirements engineering, vol. 8, pp. 149-160, 2003.

M. Romero, A. VizcaÃ-no, and M. Piattini, "Teaching requirements elicitation within the context of global software development," presented at Computer Science (ENC), 2009 Mexican International Conference on, 2009.

N. Sabahat, F. Iqbal, F. Azam, and M. Y. Javed, "An iterative approach for global requirements elicitation: A case study analysis," presented at Electronics and Information Engineering (ICEIE), 2010 International Conference On, 2010.

S. Neetu Kumari and A. S. Pillai, "A survey on global requirements elicitation issues and proposed research framework," presented at Software Engineering and Service Science (ICSESS), 2013 4th IEEE International Conference on, 2013.

G. N. Aranda, A. VizcaÃ-no, and M. Piattini, "A framework to improve communication during the requirements elicitation process in GSD projects," Requirements engineering, vol. 15, pp. 397-417, 2010. 
M. Niazi and S. Shastry, "Critical Success Factors for the Improvement of Requirements Engineering Process.," presented at Software Engineering Research and Practice, 2003

J. Iqbal, R. Ahmad, M. H. Nizam, M. Nasir, and M. A. Noor, "Significant Requirements Engineering Practices for Software Development Outsourcing," presented at Software Engineering Conference (ASWEC), 2013 22nd Australian, 2013.

S. Hansen and K. Lyytinen, "Challenges in contemporary requirements practice," presented at System Sciences (HICSS), 2010 43rd Hawaii International Conference on, 2010.

M. Kauppinen, M. Vartiainen, J. Kontio, S. Kujala, and R. Sulonen, "Implementing requirements engineering processes throughout organizations: success factors and challenges," Information and Software Technology, vol. 46, pp. 937-953, 2004.

N. M. Minhas and A. Zulfiqar, "An Improved Framework for Requirement Change Management in Global Software Development," Journal of Software Engineering and Applications, vol. 7, pp. 779, 2014.

A. Ahmad and H. Khan, "The Importance of Knowledge Management Practices in Overcoming the Global Software Engineering Challenges in Requirements Understanding," Blekinge Institute of Technology, Ronneby, Sweden, pp. 169, 2008

G. N. Aranda, A. VizcaÃ-no, and M. Piattini, "Analyzing and Evaluating the Main Factors that Challenge Global Software Development," Open Software Engineering Journal, vol. 4, pp. $14-25,2010$

D. Savio, P. Anitha, A. Patil, and O. Creighton, "Visualizing requirements in distributed system development," presented at Requirements Engineering for Systems, Services and Systems-of-Systems (RES4), 2012 IEEE Second Workshop on, 2012.

H. K. Edwards and V. Sridhar, "Analysis of the effectiveness of global virtual teams in software engineering projects," presented at System Sciences, 2003. Proceedings of the 36th Annual Hawaii International Conference on, 2003.

M. Heindl and S. Biffl, "Risk management with enhanced tracing of requirements rationale in highly distributed projects," presented at Proceedings of the 2006 international workshop on Global software development for the practitioner, 2006

B. Berenbach, "Impact of organizational structure on distributed requirements engineering processes: lessons learned," presented at Proceedings of the 2006 international workshop on Global software development for the practitioner, 2006.

E. MacGregor, Y. Hsieh, and P. Kruchten, "Cultural patterns in software process mishaps: incidents in global projects," ACM SIGSOFT Software Engineering Notes, vol. 30, pp. 1-5, 2005.

D. $\AA$ mite, "Requirements management in distributed projects," Journal of Universal Knowledge Management, vol. 1, pp. 69-76, 2006.

C. Ebert, B. K. Murthy, and N. N. Jha, "Managing risks in global software engineering: principles and practices," presented at Global Software Engineering, 2008. ICGSE 2008. IEEE International Conference on, 2008.

S. V. Shrivastava and U. Rathod, "Categorization of risk factors for distributed agile projects," Information and Software Technology, vol. 58, pp. 373-387, 2015.

Z. Haag, R. Foley, and J. Newman, "Software process improvement in geographically distributed software engineering: an initial evaluation," presented at EUROMICRO 97. New Frontiers of Information Technology., Proceedings of the 23rd EUROMICRO Conference, 1997.

M. Korkala and P. Abrahamsson, "Communication in distributed agile development: A case study," presented at Software Engineering and Advanced Applications, 2007. 33rd EUROMICRO Conference on, 2007.

I. H. de Farias Junior, R. R. de Azevedo, H. P. de Moura, and D. S. Silva, "Elicitation of Communication Inherent Risks in Distributed Software Development," presented at Global Software Engineering Workshops (ICGSEW), 2012 IEEE Seventh International Conference on, 2012.

M. Pichler, H. Rumetshofer, and W. Wahler, "Agile requirements engineering for a social insurance for occupational risks organization: A case study," presented at Requirements Engineering, 14th IEEE International Conference, 2006.

J. M. Bhat, M. Gupta, and S. N. Murthy, "Overcoming requirements engineering challenges: Lessons from offshore outsourcing," Software, IEEE, vol. 23, pp. 38-44, 2006.

S. Jalali and C. Wohlin, "Agile Practices in Global Software Engineering-A Systematic Map.," presented at ICGSE, 2010.

S. Ghaisas, "A Method for Identifying Unobvious Requirements in Globally Distributed Software Projects.," presented at Software Engineering (Workshops), 2009.

S. A. Kumar and T. A. Kumar, "Study the Impact of Requirements Management Characteristics in Global Software Development Project: An Ontology Based Approach," International Journal of Software Engineering and Application, vol. 2, 2011.

J. Hanisch and B. Corbitt, "Impediments to requirements engineering during global software development," European Journal of Information Systems, vol. 16, pp. 793-805, 2007.

R. Lai and N. Ali, "A Requirements Management Method for Global Software Development," Advances in Information Sciences (AIS), vol. 1, pp. 38-58, 2013.

R. Prikladnicki and J. Audy, "Requirements engineering in global software development: Preliminary findings from a case study in a SW-CMM context," Proc. of the 5th SIMPROSâ€"Simp $\tilde{A}^{3}$ sio Internacional de Melhoria de Processo de Software, Pernambuco, 2003.

T. Illes-Seifert, A. Herrmann, M. Geisser, and T. Hildenbrand, "The Challenges of Distributed Software Engineering and Requirements Engineering: Results of an Online Survey," presented at WORKSHOP P, 2007.

A. K. Thurimella and T. Wolf, "Issue-based variability Modeling," presented at International Conference on Global Software Engineering, Munique, Alemanha, 2007.

I. Kwan, D. Damian, and S. Marczak, "The effects of distance, experience, and communication structure on 
requirements awareness in two distributed industrial software projects," presented at Global Requirements Engineering Workshop (GREWâ€ ${ }^{\mathrm{TM}} 07$ ), in Proc. of International Conference on Global Software Engineering (ICGSE 2007), Munich, Germany, 2007.

M. Heindl and S. Biffl, " Risk management with enhanced tracing of requirements rationale in highly distributed projects," presented at Proceedings of the 2006 international workshop on Global software development for the practitioner, 2006

V. Mikulovic and M. Heiss, "How do I know what I have to do?: the role of the inquiry culture in requirements communication for distributed software development projects," presented at Proceedings of the 28th international conference on Software engineering, 2006.

F. Calefato, D. Damian, and F. Lanubile, "Computer-mediated communication to support distributed requirements elicitations and negotiations tasks," Empirical Software Engineering, vol. 17, pp. 640-674, 2012.

S. Dorairaj, J. Noble, and P. Malik, "Effective communication in distributed Agile software development teams," in Agile Processes in Software Engineering and Extreme Programming: Springer, 2011, pp. 102-116.

J. M. C. de Gea, J. n. Nicol $\tilde{A}_{i s}$, J. L. F. n. Alem $\tilde{A}_{i n}$, A. Toval, A. Vizca $\tilde{A}-n o$, and C. Ebert, "Reusing requirements in global software engineering," in Managing requirements knowledge: Springer, 2013, pp. 171-197.

E. Bjarnason, "Distances between requirements engineering and later software development activities: a systematic map," in Requirements Engineering: Foundation for Software Quality: Springer, 2013, pp. 292-307.

P. Liang, P. Avgeriou, and V. Clerc, "Requirements reasoning for distributed requirements analysis using semantic wiki," presented at Global Software Engineering, 2009. ICGSE 2009. Fourth IEEE International Conference on, 2009.

A. Lamersdorf and J. r. MÃ¹/4nch, "A multi-criteria distribution model for global software development projects," Journal of the Brazilian Computer Society, vol. 16, pp. 97115,2010

R. Narayanaswamy and R. M. Henry, "Effects of culture on control mechanisms in offshore outsourced IT projects," presented at Proceedings of the 2005 ACM SIGMIS CPR conference on Computer personnel research, 2005.

S. Sakthivel, "Managing risk in offshore systems development," Communications of the ACM, vol. 50, pp. 6975, 2007.

V. Dalberg, E. Angelvik, D. R. Elvekrok, and A. K. Fossberg, "Cross-cultural collaboration in ICT procurement," presented at Proceedings of the 2006 international workshop on Global software development for the practitioner, 2006.

S. Overhage, O. Skroch, and K. Turowski, "A Method to Evaluate the Suitability of Requirements Specifications for Offshore Projects," Business \& Information Systems Engineering, vol. 2, pp. 155-164, 2010.

J. D. Herbsleb and A. Mockus, "An empirical study of speed and communication in globally distributed software development," Software Engineering, IEEE Transactions on, vol. 29, pp. 481-494, 2003.
L. Lopes, R. Prikladnicki, J. L. N. Audy, and A. Majdenbaum, "Distributed Requirements Specification: Minimizing the Effect of Geographic Dispersion.," presented at ICEIS (3), 2004.

E. Hossain, M. A. Babar, H.-y. Paik, and J. Verner, "Risk identification and mitigation processes for using scrum in global software development: A conceptual framework," presented at Software Engineering Conference, 2009. APSEC'09. Asia-Pacific, 2009.

L. Karlsson, Â. s. G. Dahlstedt, B. r. Regnell, J. N. och Dag, and A. Persson, "Requirements engineering challenges in market-driven software developmentâ€"An interview study with practitioners," Information and Software technology, vol. 49 , pp. 588-604, 2007.

S. V. Shrivastava and U. Rathod, "Risks in distributed agile development: A review," Procedia-Social and Behavioral Sciences, vol. 133, pp. 417-424, 2014.

D. M. n. Fern $\tilde{A}_{i}$ ndez and S. Wagner, "Naming the pain in requirements engineering: A design for a global family of surveys and first results from Germany," Information and Software Technology, vol. 57, pp. 616-643, 2015.

A.-M. SÃ derberg, S. Krishna, and P. BjÃ rn, "Global software development: commitment, trust and cultural sensitivity in strategic partnerships," Journal of International Management, vol. 19, pp. 347-361, 2013.

B. Javed and S. S. Minhas, "Process Support for Requirements Engineering Activities in Global Software Development: A Literature Based Evaluation," presented at Computational Intelligence and Software Engineering (CiSE), 2010 International Conference on, 2010. 